\title{
Robust dynamical pattern formation from a multifunctional minimal genetic circuit
}

\author{
Guillermo Rodrigo 1,2, Javier Carrera1,2,3, Santiago F Elena',4 and Alfonso Jaramillo*2,5
}

\begin{abstract}
Background: A practical problem during the analysis of natural networks is their complexity, thus the use of synthetic circuits would allow to unveil the natural mechanisms of operation. Autocatalytic gene regulatory networks play an important role in shaping the development of multicellular organisms, whereas oscillatory circuits are used to control gene expression under variable environments such as the light-dark cycle.

Results: We propose a new mechanism to generate developmental patterns and oscillations using a minimal number of genes. For this, we design a synthetic gene circuit with an antagonistic self-regulation to study the spatio-temporal control of protein expression. Here, we show that our minimal system can behave as a biological clock or memory, and it exhibites an inherent robustness due to a quorum sensing mechanism. We analyze this property by accounting for molecular noise in an heterogeneous population. We also show how the period of the oscillations is tunable by environmental signals, and we study the bifurcations of the system by constructing different phase diagrams.
\end{abstract}

Conclusions: As this minimal circuit is based on a single transcriptional unit, it provides a new mechanism based on post-translational interactions to generate targeted spatio-temporal behavior.

\section{Background}

Synthetic Biology aims to engineer genetic networks with defined dynamics [1]. For this, it usually relies on the use of design principles derived from the analysis of natural genetic networks. Those networks are large and complex systems with many unknown interactions that can dramatically affect the system dynamics. Then, for a complete understanding of the mechanisms underlying gene networks it is valuable the engineering of synthetic circuits that have a minimal complexity. In addition, such small circuits would allow the modular design of complex hierarchical structures with targeted spatial and temporal behaviors. However, even the design of small circuits with existing genetic components is very challenging due to the lack of enough parameters to fine-tune the system. In fact, the use of properly characterized genetic components favors an accurate prediction of the dynamics of an in vivo implemented circuit [2-5]. The extreme case being the design of a genetic network composed of a single transcriptional unit showing a specified spatio-temporal

* Correspondence: alfonso.jaramillo@polytechnique.fr

2 Synth-Bio group. Epigenomics Project, Genopole-Université Évry Val d'Essonne-CNRS UPS3201, 91034 Évry Cedex, France

Full list of author information is available at the end of the article dynamics. As all the protein concentrations shall be coupled, it is very difficult to have a non-trivial dynamics unless the time scales of protein interactions and of cellto-cell communication are conveniently coupled.

In higher organisms, development results from the coordinated action of thousands of genes at any moment during the cell cycle. However, small regulatory circuits control the execution of genetic programs by triggering cell differentiation according to spatial patterns [6]. These patterns result from gradients of signaling molecules, which diffuse in the medium and are sensed at each moment by the cell circuitry. Quantitative models based on reaction-diffusion equations have been successfully applied to understand the principles of organism's development [7-9]. Furthermore, synthetic patterns have been previously engineered in bacteria [10] and flies [11]. However, genetic systems with defined spatial and temporal behavior have not been artificially constructed yet. In such a synthetic system, the fate of every cell within the population could be controlled, for instance, by oscillators working in a specific manner in response to spatial location or by the state of an internal memory. It is of particular interest to apply the same design principles underlying naturally occurring molecular clocks, where 
rythmicity is mainly based on negative feedback loops [12], to the in vivo engineering of synthetic oscillatory circuits $[13,14]$.

The simplest imaginable genetic circuit consists in a single operon with a feedback loop. On the one hand, negative autoregulation promotes robustness [15], but it can also cause oscillations if the process introduces a delay [16-18]. On the other hand, positive autoregulation yields bistability [19]. By combining both structures, we have designed and analyzed theoretically a synthetic genetic circuit with a minimal transcription structure exhibiting multifunctionality (Fig. 1a). We present a mathematical model at the molecular level based on differential equations for the synthetic self-regulated transcription circuit. The system shows oscillatory and bistable behaviors, together with intrinsic robustness through a quorum sensing (QS) mechanism (Fig. 1b) that allows for cellular synchronization [20,21]. The system, which is expressed from plasmids, consists of two transcription factors (TFs) responding to two different chemicals. Thus, we perform spatio-temporal simulations showing different dynamic pattern formation depending on the initial environment.

\section{Results and Discussion}

The system, a single transcriptional unit, consists in a combinatorial promoter, lactose-luciferase, which controls the expression of two TFs LacI and LuxR, and the enzyme LuxI (see Methods for further details). Being all the concentrations of protein species proportional, it would make a priori especially difficult our targeted dynamics. Fortunately, we can still have a rich dynamics

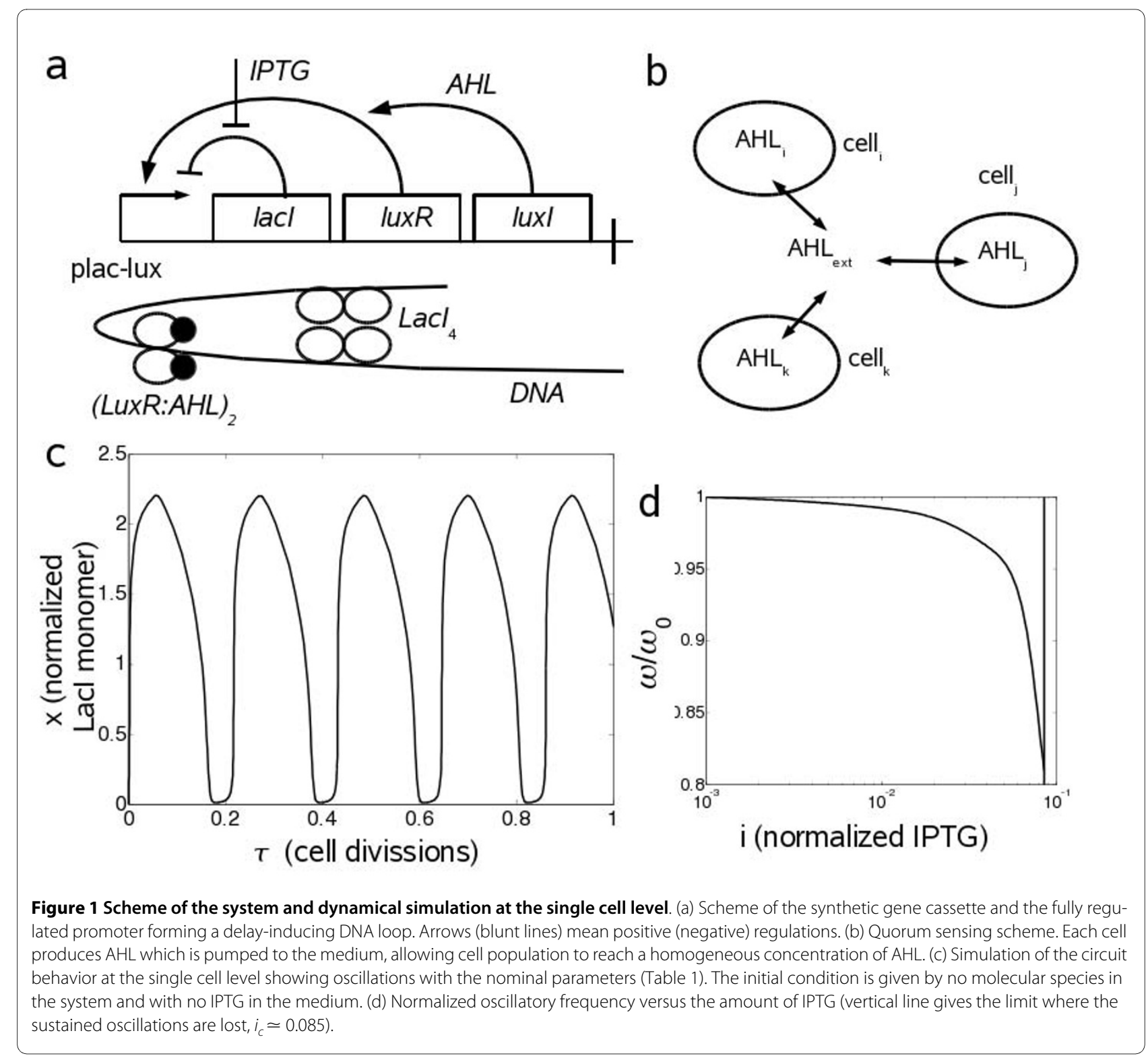


at single cell owed to the suitable design of molecular interactions (multimerization and binding events). Furthermore, this model is coupled to a population model, where cell-to-cell communication introduces the spatial dimension. Usually the models including the spatial dimension require the use of several genes with uncoupled dynamics. Here we will show that a dynamical pattern behavior can be generated by using genes expressing the same concentration of proteins (up to a proportionality factor).

\section{Multifunctional behavior}

Using experimentally measured parameter values (Table 1), we simulate the dynamic behavior of the system (Fig. 1c). The period of the oscillations is about 20 min, which is 5 -fold shorter than the cell doubling period $\left(\tau_{0}=100\right.$ $\mathrm{min}$ ). This period can be environmentally tuned without genetic modifications of the circuit. We show the evolution of the frequency of oscillations $(\omega)$, normalized by the nominal one from Fig. 1c $\left(\omega_{0}\right)$, for different concentrations of IPTG (Fig. 1d), resulting in a losing of oscillations at high levels of IPTG $\left(i>i_{c}\right)$ due to the inactivation of the negative loop. In addition, higher values of the enzymatic degradation coefficient $(\delta)$ yield higher $\omega$ because of the rapidity of the transients (data not shown).

We analyze the functional sensitivity of the system. In Fig. 2a we plot the phase diagram showing the Hopf bifurcation. The delay and the nonlinearity of the repressive loop enhance the oscillatory behavior. Contrarily, at high concentration of IPTG the system is stable and it can reach bistability according to the bifurcation diagram shown in Fig. 2b. Furthermore, we study the sensitivity of the dynamical behavior to different kinetic parameters of the model (Fig. 3). We have selected the most relevant ones to study the dynamical properties of steady states reached. We show a robust oscillatory region, which would give strong chances for a successful mode of action. Certainly, the ranges for the appropriate kinetic parameter values are sufficiently large to ensure a potential biological implementation.

Firstly, we have carried out an analysis of the effect of the transcription and degradation terms. These terms are proportional to the plasmid copy number $(C)$ and the enzymatic degradation coefficient $(\delta)$, respectively. On the one hand, in Fig. 3a we can observe oscillatory and bistability regions. High values of $C$ yields monostability. However, intermediate values yields oscillations at low levels of IPTG, whereas low values yields bistability at high levels of IPTG. Importantly, the external addition of high amounts of AHL enhances the oscillatory region in detriment to bistability (Fig. 3c). This fact allows a transition between oscillations and bistability using IPTG and AHL. To better illustrate this transition, we have performed a stability analysis of the circuit, with $C=10$, using IPTG and external AHL as control parameters (Fig. 4). The map shows four different regions: bistable at high levels of IPTG and low of external AHL, oscillatory at low IPTG and high external AHL, and monostable in the other two cases. On the other hand, the circuit is monostable for low values of $\delta$, oscillatory for intermediate values and low levels of IPTG, and bistable for high values and high levels of IPTG (Fig. 3b). Analogously to the previous case, the external addition of AHL enhances the oscillatory region for high values of $\delta$, thus allowing a transition between the oscillatory and bistability regimes (Fig. 3d). Additionally, in Fig. 3e, we show how the delay $(T)$ is necessary to reach the oscillatory regime. In that sense, the minimal required delay is about 4 minutes (for the nominal values of the kinetic parameters shown in Table 1). In this work we have considered a delay of 5.5 minutes, based on the estimation of the unlooping kinetic constant in $0.18 \mathrm{~min}^{-1}$ [22]. According to Fig. 3f, cooperative binding between $\mathrm{Lacl}_{4}$ and (LuxR:AHL) ${ }_{2}(\Omega>1)$ enhances the oscillatory regime, whereas competitive

Table 1: Kinetic parameters used in the spatiotemporal transcription model.

\begin{tabular}{|c|c|c|c|}
\hline Parameter & Ref. & Parameter & Ref. \\
\hline$a=125 \mathrm{nM} / \mathrm{min}$ & [35] & $\theta_{Y}=10 \mathrm{nM}$ & [10] \\
\hline$\Lambda=75 \mathrm{nM}$ & [26] & $\theta_{X}=5 \mathrm{nM}$ & {$[22]$} \\
\hline$\delta=300 \mathrm{nM} / \mathrm{min}$ & {$[26]$} & $\theta_{l}=15 \mu \mathrm{M}$ & [23] \\
\hline$L_{0}=4.1$ & [23] & $T=5.5 \mathrm{~min}$ & [22] \\
\hline$K_{1}=100 \mathrm{nM}$ & [2] & $K_{2}=10 \mathrm{nM}$ & [2] \\
\hline$K_{3}=55 \mathrm{nM}$ & * & $K_{4}=300 \mathrm{nM}$ & $*$ \\
\hline$\kappa=10 \mathrm{nM} / \mathrm{min}$ & [32] & $\eta=2000 \mathrm{~min}^{-1}$ & [33] \\
\hline$\delta_{A}=0.002 \mathrm{~min}^{-1}$ & [30] & $\lambda_{A}=1 \mathrm{~min}^{-1}$ & [30] \\
\hline$N_{m}=10^{5}$ cells $/ \mathrm{nL}$ & * & $D=10^{-3} \mathrm{~mm}^{2} / \mathrm{min}$ & [10] \\
\hline
\end{tabular}

In this work, we consider $C=50, \sigma=1, \tau_{0}=100 \mathrm{~min}^{-1}, \varphi=100$, and $\Omega=1$. ${ }^{*}$ This value was estimated from the literature. 

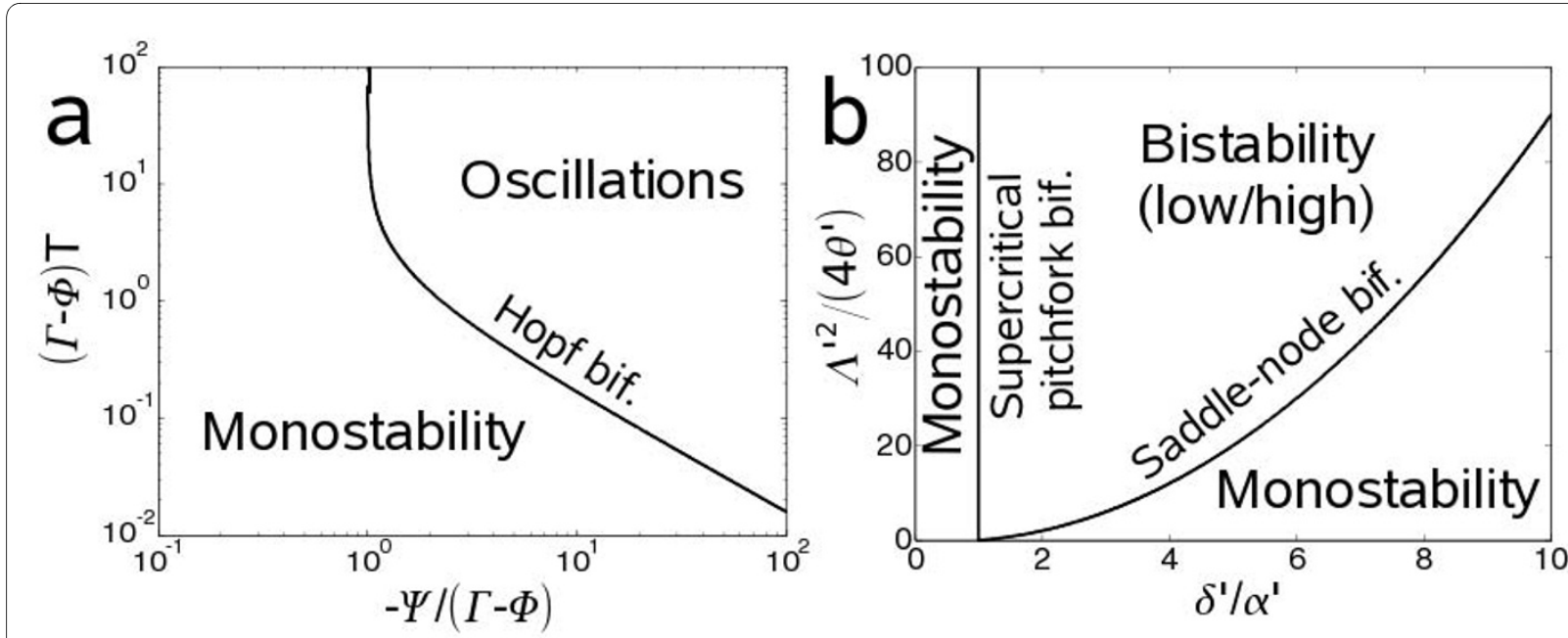

Figure 2 Stability analysis of the dynamic genetic circuit at the single cell level. (a) Oscillatory region of the system at very low levels of IPTG $(i=$ 0 ). $\Phi, \Psi$, and $\Gamma$ are defined in the main text. The bifurcation for bistability is at $-\Psi /(\Gamma-\Phi)=-1$ (not shown in the Figure). (b) Bistability region of the system at very high levels of IPTG $(i>>1)$ and external AHL $(a>>1)$. To plot (b) we have considered a simplified model (see Methods).

binding $(\Omega<1)$ makes the system stable. In the natural case of $\mathrm{LacI}_{4}$ and $\mathrm{CRP}_{2}$ :cAMP, the value of $\Omega$ was estimated in 10.3 [23]. Moreover, in Figs. 3g, h we analyze the protein-DNA binding coefficients for the two TFs of the circuit $\left.\left(\theta_{X} \text { for } \mathrm{LacI}_{4} \text { and } \theta_{Y} \text { for (LuxR:AHL) }\right)_{2}\right)$. They have antagonistic effects for the dynamical behavior of the circuit, that is, lower values of $\theta_{X}$ and higher of $\theta_{Y}$ enhance oscillations. This fact increases the experimental chances to implement such a circuit, since LacI is considered a strong repressor and the activation capacity of LuxR will not be decisive for the proper function of the circuit.

Interestingly, our circuit does not need further genetic manipulation to change the behavior regime. Environmental signals control the dynamics leading a fully tunable circuit, since by varying the concentration of IPTG and AHL we can change the dynamical regime. According to our model, this minimal genetic unit can display complex dynamics. The integration in a single circuit of the ability of oscillating and having memory may have important applications in Synthetic Biology.

\section{Synchronization of oscillations}

As we have a population of biological clocks coupled through a diffusive molecule, it is very interesting to analyze the onset of synchronization of such oscillators. We address this at local level, without considering spatial features. The equilibrium between the extra and intracellular species is much faster than their diffusion in the medium, thus it is reasonable to uncouple these two transport mechanisms to study the synchronization by QS. Moreover, we have performed such simulations with no IPTG in the medium. Firstly, we have considered a heterogeneous population where each cell has a different number of plasmidic copies $(C)$ and enzymatic degradation coefficient $(\delta)$. Thus, these parameters are assumed Gaussian random numbers, $C=N(50,10)$ and $\delta=N(300$, 10). Poisson distributions give equal results (data not shown). Following the frequency histograms shown in Figs. 5a, b, QS provides a population synchronization as the variance of the distribution with QS compared to the case without QS is significantly smaller. This fact suggests that genetic mutations that eventually affect the kinetic properties of the circuit and occur in all single individuals within the population, but in an uncorrelated way, are compensated in terms of population. Importantly, synchronization by QS relies on symmetrical distributions around a nominal value. However, QS will fail to synchronize when mutations always affect the model parameters in the same sense.

Secondly, we have introduced an intrinsic white noise to account for the stochasticity raised from the small number of molecules. It is important to notice that this noise is always present in a discrete system of molecules, although at high number of molecules it is usually neglected. We have not accounted for external sources of noise. Power spectral density (PSD) analysis has been performed showing that QS enhances regular oscillations, since in that case there is one frequency with a PSD significantly higher (Figs. 5c, d). The global effect of AHL together with the different temporal scales in the system, first producing the activator then the repressor with a delay, enables to sustain in time the oscillatory behavior of the whole population.

\section{Spatio-temporal patterns}

The fact of having an oscillating circuit allows the obtaining of patterns with dynamical behavior. The possibility 

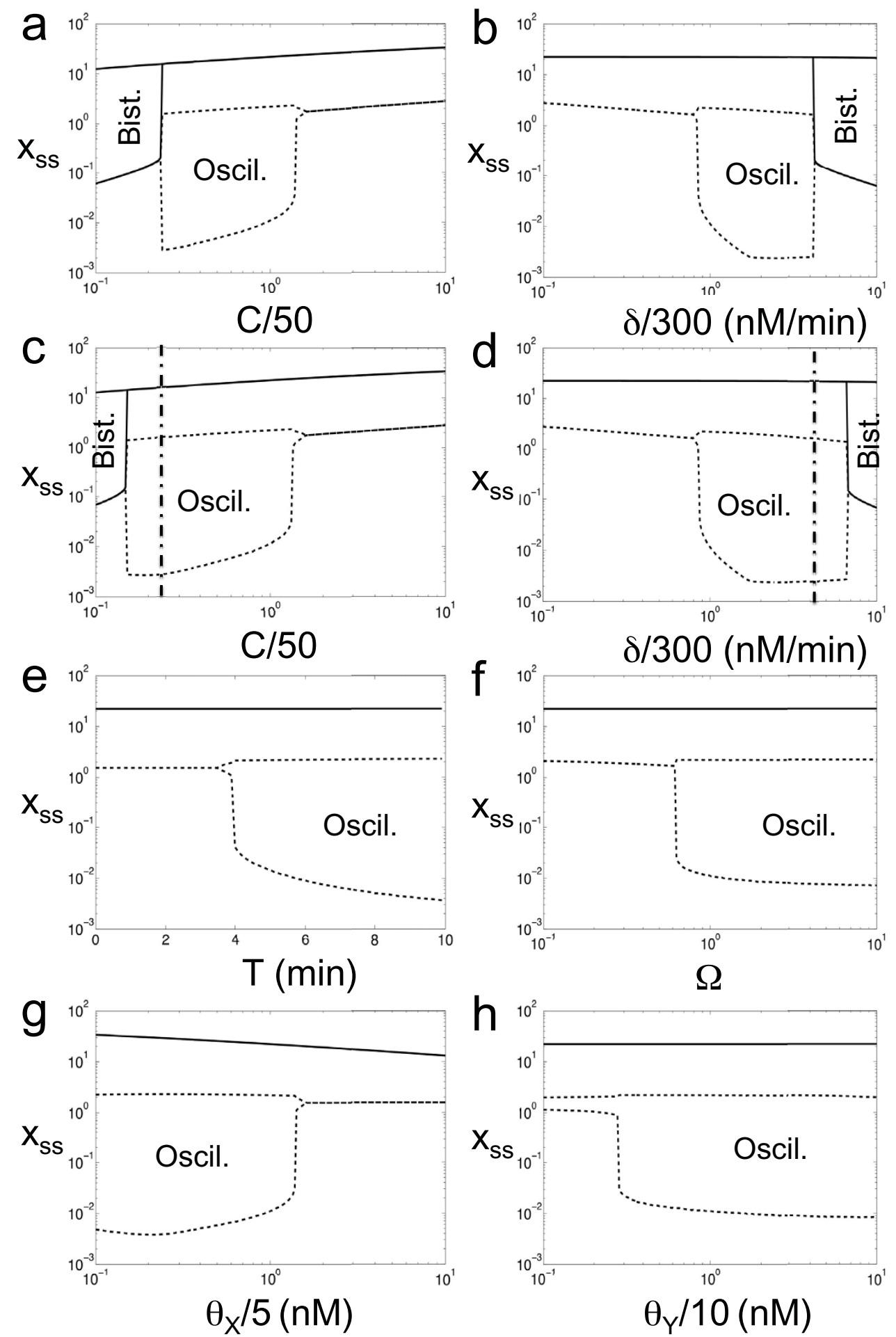

Figure 3 Sensitivity analysis of the circuit for the most outstanding parameters. The rest of the parameter values are shown in Table 1. We plot the steady state(s) $\left(x_{\text {ss }}\right)$ at very high levels of IPTG, $i=10^{3}$ (solid lines), and with no IPTG (dashed lines). Bifurcation of solid lines indicates the bistability limit condition. Bifurcation of dashed lines represents the oscillatory limit point, where we plot the maximal and minimal values of the oscillatory dynamics. In c, d AHL is externally introduced at very high levels. 


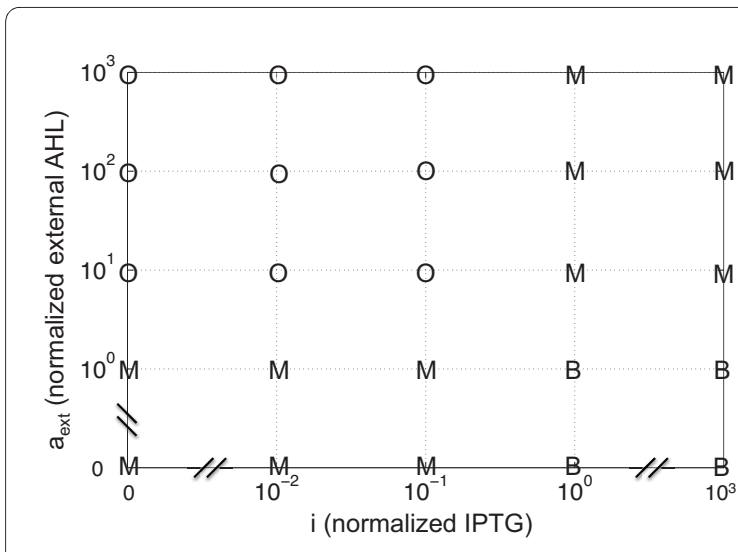

Figure 4 Stability analysis of the circuit as a function of IPTG and external AHL (assumed constant). Herein, we consider a low plasmid copy number $(C=10)$. We plot the dynamical regimes of the circuit ( $\mathrm{M}$, monostability; $\mathrm{B}$, bistability; $\mathrm{O}$, oscillations) for different values of the external inducers.

of producing waves in genetic systems opens the door to the development of new types of tissue engineering that could adapt to the environment. On the one hand, In Figs. 6a, b we show the spatio-temporal pattern evolution triggered by IPTG. An initial stable state of high LacI is reached quickly and it propagates to neighboring cells outwards in radial direction as IPTG diffuses. At large radius we have low IPTG that gives oscillations until the concentration of IPTG reaches the critical level $\left(i_{c}\right)$. This composed structure can be viewed as a spatial pulse that filters oscillations into constant signals. On the other hand, in Figs. 6c, d AHL is externally introduced at high levels to generate patterns that change with time as AHL diffuses. The spatial structure of concentric rings dynamically changes by varying the protein expression level at each point and adding more rings. At low radius, there is a monomodal transition from low to high expression levels, indicating that the amount of AHL is above certain threshold that controls gene expression in that region. By assuming a non-homogeneous diffusion, we could generate non-symmetrical structures. Each cell carries an oscillator whose period depends on the spatial location and time.

The in vivo implementation of such a circuit and its characterization could be the matter of further work, beyond the scope of this study. Experimentally, a fluorescent protein such as GFP could be inserted into the operon as reporter. Time-lapse microscopy could be used to visualize fluorescence in solid-phase in a scale of various $\mathrm{cm}$ in space and tens of generations in time [10],
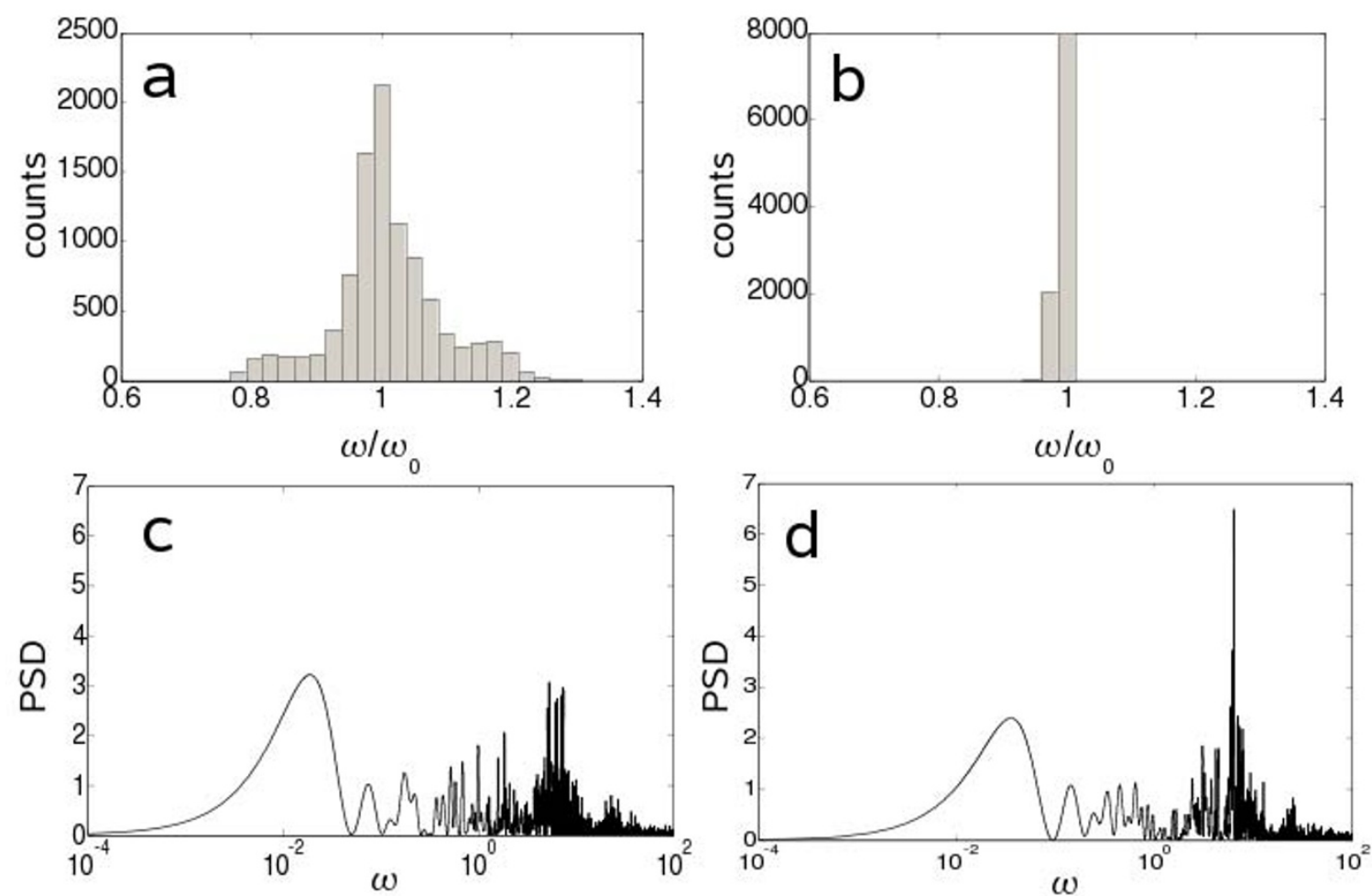

Figure 5 Frequency histogram of the system response (a) without and (b) with quorum sensing. To plot those histograms, Cand $\delta$ are assumed to be Gaussian random numbers with mean 50 and 300 respectively and standard deviation 10 . Power spectral density (PSD) analysis by considering a white noise (c) without and (d) with quorum sensing. Figures are obtained at very low levels of IPTG $(i=0)$. 


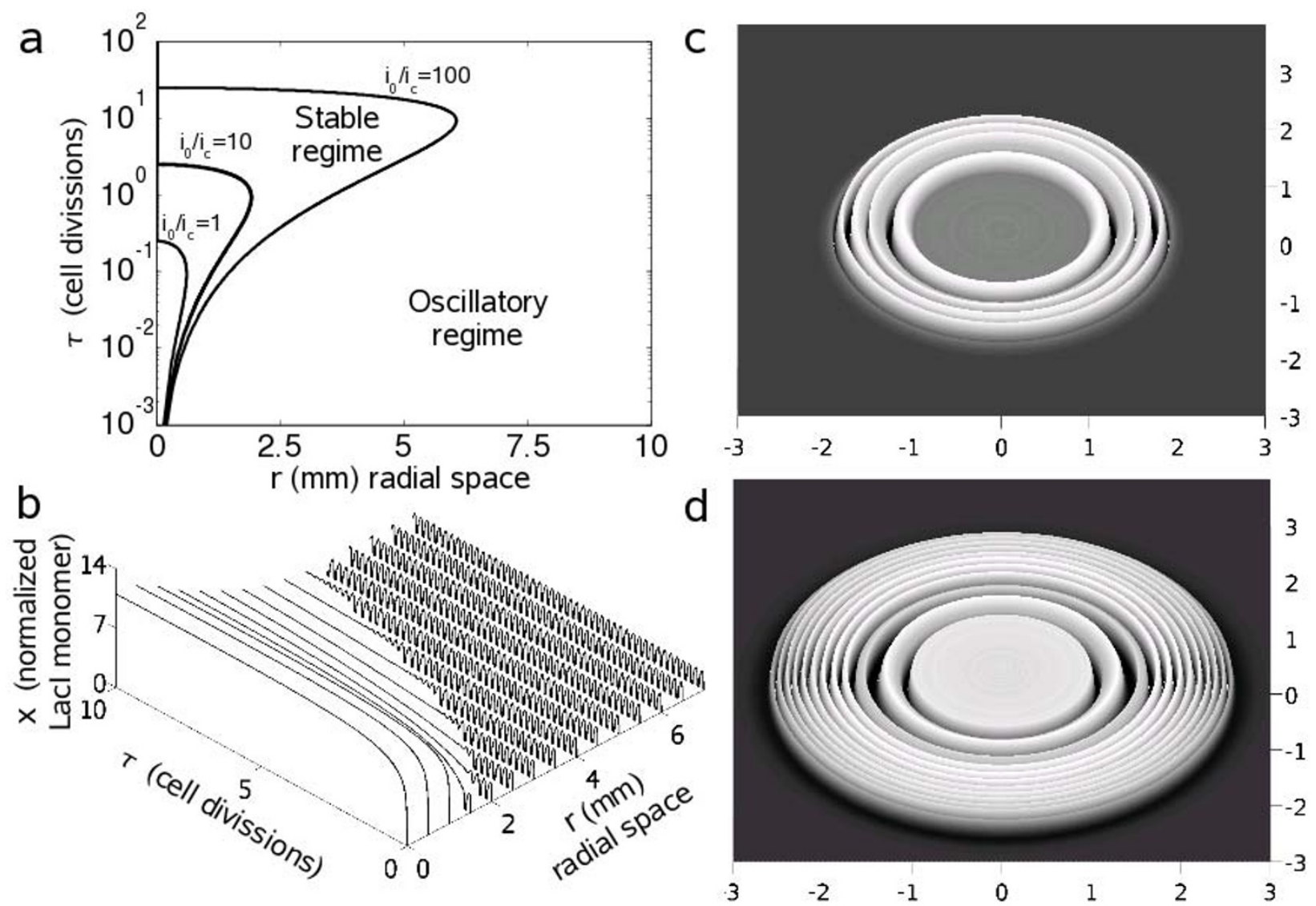

Figure 6 Dynamical pattern formation. (a) Spatio-temporal diagram showing the different regimes of operation (front given by $r_{c}$ ). (b) Dynamical behavior in response to an external signal of IPTG $\left(/(r=0, \tau=0)=10^{6} \mathrm{nM}\right)$. (c and d) Spatial plot at different times $(\tau=1$ and $\tau=3)$ showing the generated patterns when AHL is externally introduced but not produced $(K=0)$ nor degraded $\left(\lambda_{A}=0\right)$ into the cells $\left(A(r=0, \tau=0)=10^{4} \mathrm{nM}\right)$. We use a white/black scale denoting high/low concentrations.

which would be sufficient to observe the dynamical patterns. Even though, this could also be tested readily with the use of microfluidic devices. In that way, the experimental work would help to validate or refine the mathematical model.

\section{Conclusions}

In this manuscript, we have shown for the first time the design of a single trancriptional unit with a post-translational dynamics that couples spatial and temporal scales to generate dynamical spatial structures. We have followed a model-based design approach to obtain a minimal genetic unit with multiple functionalities and displaying certain homeostasis to both environmental and mutational perturbations, since external fluctuations or variations in kinetic parameters are compensated due to QS. We have relied on nonlinear dynamics and stochastic modeling to analyze our system under biological noise. We have provided a new mechanism able to switch from oscillatory to bistable regimes using an external inducer and to produce complex spatio-temporal patterns by using a single transcriptional unit. In addition, this sort of small functional modules could be hierarchically assembled to generate more complex systems [24]. That these simple units are able to generate such a complex behavior provides new avenues to understand natural genetic circuits by designing synthetic minimal systems. The bottleneck in the construction of a synthetic network consists in the number of independent transcriptional units, as transcription carries the largest source of intrinsic and extrinsic noises [25]. Although a system consisting in a single operon would be very appealing, it may have serious problems due to its minimalism unless the system is properly designed. In the past, most authors have used at least two operons to construct systems with oscillatory behavior or producing a patterning. Although for the former, it was already known several examples of oscillatory circuits consisting in a single operon. That our minimal circuit can display such a rich behavior highlights the fact that rational design tech- 
niques take advantage of engineering principles for constructing genetic circuits with specified functions.

\section{Methods}

\section{Dynamics at the single cell level}

A synthetic lactose-luciferase (lac-lux) promoter controls the transcription of the lac repressor LacI $(X)$, the activator LuxR $(Y)$ and the enzyme LuxI $(Z)$. Here, we consider the bacterium Escherichia coli as cellular chassis, and all proteins are assumed to be ssrA-tagged for enzymatic degradation [26], which for a zeroth-order kinetics enhances robustness of oscillations [27]. In addition, the chemical IPTG $(I)$ acts as inducer and binds to LacI inhibiting its repressive effect; the chemical AHL $(A)$ is required by LuxR to activate transcription. The active form of LacI is a tetramer $\left(X_{4}\right)$, whereas for LuxR it consists of a dimmer of the complex LuxR-AHL $\left((Y: A)_{2}\right)$. In addition, repression by LacI tetramer induces a DNA loop in the promoter region (1a) [28], which may introduce a delay $(T)$ into the system. The processes of transcription, translation, folding and multimerization could also induce a delay, but this is neglected in this work. Indeed, transcription in eukaryotes dictates a delay because of splicing [16]. However, this is not the case in prokaryotes. Herein, we assume that the reaction between two tetramers for making the DNA loop is not reversible, given that the looping structure remains even for low levels of LacI [22].

Furthermore, here we assume a fast mRNA dynamics (as the quasi-steady state is roughly reached in $5 \mathrm{~min}$ ) [29]. The total concentrations of the three proteins $(X, Y$ and $Z$ ) remain proportional, then we reduce the model to a single variable. The dynamics of the regulatory model is given by

$$
\frac{d X}{d t}=C \alpha f\left(X_{4},(Y: A)_{2}, I\right)-\left(\mu+\frac{\delta}{\Lambda+(1+2 \sigma) X}\right) X
$$

where $C$ is the plasmid copy number, $\alpha$ the nominal transcription/translation rate, $\delta$ and $\Lambda$ the kinetic constants of the ClpXP protease, $\mu$ the cell growth rate, and $\sigma$ the ratio activator/repressor $(X=\sigma Y=\sigma Z$, being $(1+2 \sigma) X$ the total protein amount). LacI-mediated DNA loop enhances the autorepression. This is incorporated into the model by introducing an additional repressive term proportional to a looping constant $\left(L_{0}\right)$. In case of no loop, $L_{0}=0$. Moreover, this repression can be modulated by (LuxR:AHL) ${ }_{2}$, as it occurs with $\mathrm{CRP}_{2}$ :CAMP in the natural lac promoter [23]. The functional form of the regulatory factor $f$ has been previously studied $[23,28]$ and is given by

$$
f\left(X_{4},(Y: A)_{2}, I\right)=\frac{\frac{1 / \varphi+(Y: A)_{2} / \theta_{Y}}{1+(Y: A)_{2} / \theta_{Y}}}{1+\frac{2 X_{4} / \theta_{X}}{\left(1+I / \theta_{I}\right)^{2}}+\frac{2 X_{4}(t-T) / \theta_{X}}{\left(1+I / \theta_{I}\right)^{4}} L_{0} \frac{1+\Omega(Y: A)_{2} / \theta_{Y}}{1+(Y: A)_{2} / \theta_{Y}}},
$$

where $\theta_{X}$ is the binding coefficient of $\mathrm{LacI}_{4}$-DNA, $\theta_{I}$ of LacI-IPTG ${ }_{2}, \theta_{Y}$ of (LuxR:AHL) $)_{2}$-DNA, and $1 / \phi$ accounts for the basal transcription rate. The parameter $\Omega$ accounts for the potential interaction of $\mathrm{LacI}_{4}$ and (LuxR:AHL) ${ }_{2}$ in the DNA loop. In case of no interaction, $\Omega=1$. In addition, the synthesis rate of AHL is assumed proportional to LuxI, and we consider that cells express the enzyme AiiA which degradates AHL [30]

$$
\frac{d A}{d t}=\kappa Z-\left(\delta_{A}+\lambda_{A}+\mu\right) A,
$$

where $\kappa$ is the synthesis constant of AHL by LuxI, $\delta_{A}$ the thermodynamic degradation constant of AHL, and $\lambda_{A}$ the degradation rate by AiiA.

The multimerization kinetics (at a given total cellular amount of AHL) is given by

$$
\begin{array}{r}
\frac{d X_{2}}{d t}=k_{1} X_{1}^{2}-k_{-1} X_{2}-2 k_{2} X_{2}^{2}+2 k_{-2} X_{4}, \\
\frac{d X_{4}}{d t}=k_{2} X_{2}^{2}-k_{-2} X_{4}, \\
\frac{d(Y: A)}{d t}=k_{3} Y_{1} A-k_{-3}(Y: A)-2 k_{4}(Y: A)^{2} \\
+2 k_{-4}(Y: A)_{2}, \\
\frac{d(Y: A)_{2}}{d t}=k_{4}(Y: A)^{2}-k_{-4}(Y: A)_{2}, \\
\frac{d A}{d t}=-k_{3} Y_{1} A+k_{-3}(Y: A),
\end{array}
$$

where $k_{l /-l}$ are the forward/reverse kinetic coefficients $(l$ $=1,2,3,4)$. By exploiting the different time scales in the dynamics and neglecting the amount of DNA-bound protein, we can define the dimensionless variables as $x=X_{1} /$ $\theta$ with $\theta=\left(K_{1}^{2} K_{2} \theta_{X}\right)^{1 / 4}, i=I / \theta_{I}, y=Y_{1} /\left(K_{4} \theta_{Y}\right)^{1 / 2}$, and $a$ $=A / K_{3}$. Notice that $y$ and $a$ depend on $x$. The reactions for multimerization can be assumed much faster than the ones for transcription and translation. Then the system (4) is reduced to the steady state. Being that, we obtain $X_{2}=X_{1}^{2} / K_{1}, X_{4}=X_{1}^{4} /\left(K_{1}^{2} K_{2}\right), Y: A=Y_{1} A / K_{3}$, and $(Y: A)_{2}=Y_{1}^{2} A^{2} /\left(K_{3}^{2} K_{4}\right)$, with $K_{l}=k_{-l} l k_{l}$. 
Furthermore, the total amounts of LacI and LuxR are $X$ $=X_{1}+2 X_{2}+4 X_{4}$ and $Y=Y_{1}+Y: A+2(Y: A)_{2}$ respectively. For simplicity in the notation, we denote $X$ as a function of the dimensionless variable $x$, $X=H(x)=\theta x+2 \frac{\theta^{2}}{K_{1}} x^{2}+4 \frac{\theta^{4}}{K_{1}^{2} K_{2}} x^{4}$, and $h(x)=\frac{\partial H}{\partial x}$. In addition, we assume that the total amount of AHL is approximately equal to the free one, then it turns out that $Y: A=\frac{K_{4}}{4}\left(-\left(1+\frac{K_{3}}{A}\right)+\sqrt{\left(1+\frac{K_{3}}{A}\right)^{2}+\frac{8 Y}{K_{4}}}\right)$. Being that, $Y: A=0$ when $A=0$ and $Y: A=\frac{K_{4}}{4}\left(-1+\sqrt{1+\frac{8 Y}{K_{4}}}\right)$ at very high levels of $A$. For the following, we denote $x_{T}=x(t$ $-T)$. Time is also re-scaled by cell division, $\tau=\mu_{0} t / \ln (2)=$ $t / \tau_{0}$.

Accordingly, the regulatory and degradation factors read, respectively, as

$$
\begin{gathered}
f\left(x, x_{T}, i, y, a\right)=\frac{\frac{1 / \varphi+(y a)^{2}}{1+(y a)^{2}}}{1+\frac{2 x^{4}}{(1+i)^{2}}+\frac{2 x_{T}^{4}}{(1+i)^{4}} L_{0} \frac{1+\Omega(y a)^{2}}{1+(y a)^{2}}} \\
d(x)=\frac{H(x)}{\Lambda+(1+2 \sigma) H(x)} .
\end{gathered}
$$

\section{Spectral analysis}

We define $\hat{f}_{i}\left(x, x_{T}\right)=C \alpha \frac{\tau_{0}}{h(x)} f\left(x, x_{T}, i, y(x), a(x)\right)$ and $\hat{d}(x)=\delta \frac{\tau_{0}}{h(x)} d(x)$. Then, the differential equation (1) reads

$$
\frac{d x}{d t}=\hat{f}_{i}\left(x, x_{T}\right)-\hat{d}(x)
$$

for a constant value of $i$. We denote $\Phi=\frac{\partial \hat{f}_{i}}{\partial x} \mid s s$, $\Psi=\frac{\partial \hat{f}_{i}}{\partial x_{T}} \mid$ ss and $\Gamma=\frac{\partial \hat{d}}{\partial x} \mid s s$, where these variables are evaluated in the steady state $\left(x_{s s}\right)$. The steady state satis-

fies $\hat{f}_{i}\left(x_{s s}, x_{s s}\right)-\hat{d}\left(x_{s s}\right)=0$. Being $\Delta x=x-x_{s s}$, using first-order perturbations we can write

$$
\frac{d \Delta x}{d t}=\Phi \Delta x+\Psi \Delta x_{T}-\Gamma \Delta x
$$

Thereby, the equation of eigenvalues $(\lambda)$ is $\lambda=\Phi-\Gamma+$ $\Psi e^{-\lambda T}$ [31]. Let us define the following variables $U_{1}=\frac{-\Psi}{\Gamma-\Phi}$ and $U_{2}=(\Gamma-\Phi) T$ to simplify the results of the spectral analysis. The oscillatory boundary condition is given by $\lambda=j \omega$, being $j$ the imaginary unit. Then we obtain $U_{2} \sqrt{U_{1}^{2}-1}=\arccos \left(-1 / U_{1}\right)$, the analytical equation for the Hopf bifurcation. On the contrary, the bistability boundary condition implies $\lambda=0$, then we obtain $U_{1}=-1$. To further analyze the bistability of the system, we simplify the model at high levels of IPTG and externally introduced AHL. Without lost of generality, a dynamics governed by $\dot{x}=\frac{\alpha^{\prime} x^{2}}{\theta^{\prime}+x^{2}}-\frac{\delta^{\prime} x}{\Lambda^{\prime}+x}$ captures the principal features of the system, where $\alpha^{\prime} \sim C \alpha, \theta^{\prime} \sim \sigma^{2} K_{4}$ $\theta_{Y} / \theta^{2}, \delta^{\prime} \sim \delta$, and $\Lambda^{\prime} \sim \Lambda$.

\section{Numerical integration and stochasticity}

To illustrate this point, let us consider a molecular system governed by the following general differential equation $\frac{d X}{d t}=f\left(X, X_{T}\right)-d(X)$, where $X$ is the protein amount. The transcription term is highly nonlinear and accounts for the system delay $(T)$. In our case, the function $f$ depends on both $X(t)$ and $X(t-T)$. For the enzymatic degradation, we have to notice that, as $\Lambda$ is a low value, this becomes zeroth-order for high values of $X$, while firstorder when $X$ is close to 0 .

The delay-based system can be numerically integrated following

$$
\begin{array}{r}
X(t+\Delta t)=X(t) \\
+\int_{t}^{t+\Delta t} d s \int_{t-T}^{t+\Delta t-T} d s^{\prime} f\left(X(s), X\left(s^{\prime}\right)\right) \\
-\int_{t}^{t+\Delta t} d s d(X(s)) .
\end{array}
$$

To solve this equation we use the MATLAB routine dde23.

To account for molecular noise we use the Langevin approach [25]. We introduce an intrinsic white noise into the model

$$
\begin{gathered}
\frac{d X}{d t}=f\left(X, X_{T}\right)-d(X) \\
+\sqrt{f\left(X, X_{T}\right)+d(X)} \eta(t),
\end{gathered}
$$


where $\eta(t)$ is a random fluctuation with $\langle\eta(t)\rangle=0$ and $\langle\eta$ $\left.(t) \eta\left(t^{\prime}\right)\right\rangle=\delta\left(t-t^{\prime}\right)$ (Dirac delta). This equation was solved using the specified routine and considering a constant noise in the integration interval $(\Delta t)$

$$
\begin{array}{r}
X(t+\Delta t)=X(t) \\
+\int_{t}^{t+\Delta t} d s \int_{t-T}^{t+\Delta t-T} d s^{\prime} f\left(X(s), X\left(s^{\prime}\right)\right) \\
-\int_{t}^{t+\Delta t} d s d(X(s)) \\
+\xi \int_{t}^{t+\Delta t} d s \int_{t-T}^{t+\Delta t-T} d s^{\prime} \sqrt{f\left(X(s), X\left(s^{\prime}\right)\right)+d(X(s))}
\end{array}
$$

where $\xi$ is a Gaussian-distributed random number with mean 0 and standard deviation $1 / \sqrt{\Delta t}$.

\section{Dynamics at the population level}

LuxI catalyzes the production of AHL which can be pumped to the medium facilitating a cell-to-cell communication (i.e., QS) [32]. We also account for the dynamics of the intracellular and extracellular (labeled with subscript $e$ ) concentrations of IPTG and AHL, and for the cell population $\left(N\right.$, with $\left.\mu(N)=\frac{d N}{N d t}\right)$ together with the spatial role in solid medium. The diffusion, the intracellular and the extracellular dynamics are given by

$$
\begin{array}{r}
\frac{d I}{d t}=-\eta\left(I-I_{e}\right)-\mu I, \\
\frac{d I_{e}}{d t}=N v \eta\left(I-I_{e}\right)+N v \mu I_{e}+D \nabla^{2} I_{e}, \\
\frac{d A}{d t}=\kappa Z-\eta\left(A-A_{e}\right)-\left(\delta_{A}+\lambda_{A}+\mu\right) A, \\
\frac{d A_{e}}{d t}=N v \eta\left(A-A_{e}\right)-\left(\delta_{A}-N v \mu\right) A_{e} \\
+D \nabla^{2} A_{e}, \\
\frac{d N}{d t}=\mu_{0} N\left(1-\frac{N}{N_{m}}\right),
\end{array}
$$

where $\eta$ the equilibrium constant of the membrane transport of IPTG and AHL [33], $v=V / V_{e}$ the ratio of volumes, $D$ the diffusion (assumed linear and homogeneous) coefficient, and $N_{m}$ the maximum cell capacity of the medium. Being the transport through the cell membrane fast, it turns out that $I \simeq I_{e}$ and $A_{e} \simeq Q A$ with

$$
Q=\frac{1}{1+\frac{\delta_{A}}{N \eta \eta}+\frac{\mu}{\eta}} .
$$

Since AHL is quickly degraded and $Q \simeq 1$ in a large population, we can take the quasi-steady state $A=\kappa Z / \lambda_{A}$ when AHL is not externally introduced. In addition, we neglect the movement of cells when replicating because even for $\tau=100$ this displacement would be $\sim 0.1 \mathrm{~mm}$.

Then, integrating the molecular and population models, the reaction-diffusion dynamics of the dimensionless cellular system is governed by

$$
\begin{array}{r}
\frac{d x}{d \tau}=\frac{\tau_{0}}{h(x)}\left(C \alpha f\left(x, x_{T}, i, a\right)-\delta d(x)\right), \\
\frac{d i}{d \tau}=\tau_{0} \nabla_{D}^{2} i, \\
\frac{d a}{d \tau}=\tau_{0}\left(\frac{\kappa}{K_{3}} \sigma H(x)-\lambda_{A} a+\nabla_{D}^{2} a\right),
\end{array}
$$

where the space is normalized by $D$. Solving the partial differential equation for IPTG diffusion in time and radial space $(r)$ [34], the signaling pattern reads

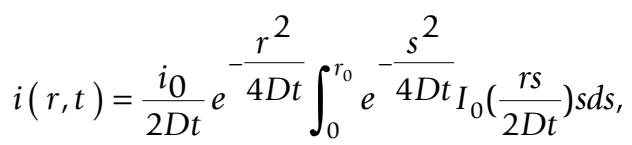

where $r_{0}$ is the radius of the initial drop of IPTG (here assumed $1 \mathrm{~mm}$ ) and $I_{0}$ is the modified Bessel function. Since for a given concentration of IPTG $\left(i_{c}\right)$ the system is not oscillatory, we obtain, using the equation (14), the spatio-temporal limit

$$
r_{c}=\sqrt{4 D t \ln \left(\frac{i_{0}}{i_{c}} \frac{r_{0}^{2}}{4 D t}\right)},
$$

where, for small values of $r_{0}\left(r_{0}<<D t\right)$, we have assumed

that $\int_{0}^{r_{0}} e^{-\frac{s^{2}}{4 D t}} I_{0}\left(\frac{r s}{2 D t}\right) s d s \simeq \frac{r_{0}^{2}}{2}$.

\section{Authors' contributions}

GR and AJ conceived this study and designed the circuit. GR developed the mathematical model and carried out the simulations. JC participated in the model construction and performed some simulations. AJ and SFE supervised the work. All authors participated in writing the manuscript. All authors read and approved the final manuscript.

\section{Acknowledgements}

We thank J. Garcia-Ojalvo for reading the manuscript and useful suggestions. This work was supported by SYNTHBIOCLOCK (CNRS Interface PhysiqueChimie-Biologie to AJ), FRM (INE20091218114 to AJ), the Generalitat Valenciana (BFPI-2007-160 to GR), the Spanish Ministry of Science and Innovation (TIN-2006-12860 to Vicente Hernandez and BFU2009-06993/BMC to SFE), the ATIGE Genopole to AJ, the Structural Funds ERDF, and the EU grants BioModularH2 (FP6-NEST-043340 to AJ), EMERGENCE (FP6-NEST-043338 to AJ) and TARPOL (FP7-KBBE-212894 to AJ). 


\section{Author Details}

IInstituto de Biología Molecular y Celular de Plantas, CSIC-UPV, 46022 Valencia, Spain, ${ }^{2}$ Synth-Bio group. Epigenomics Project, Genopole-Université Évry Val d'Essonne-CNRS UPS3201, 91034 Évry Cedex, France, 3Instituto ITACA, Universidad Politécnica de Valencia, 46022 Valencia, Spain, ${ }^{4 S a n t a ~ F e ~ I n s t i t u t e, ~}$ Santa Fe, NM 87501, USA and ${ }^{5}$ Ecole Polytechnique, Laboratoire de Biochimie, 91128 Palaiseau Cedex, France

Received: 17 November 2009 Accepted: 22 April 2010

Published: 22 April 2010

\section{References}

1. Benner SA, Sismour AM: Synthetic biology. Nat Rev Genet 2005, 6:533-543.

2. Guido NJ, Wang X, Adalsteinsson D, McMillen D, Hasty J, Cantor CR, Elston TC, Collins JJ: A bottom-up approach to gene regulation. Nature 2006, 439:856-860.

3. Ellis T, Wang X, Collins JJ: Diversity-based, model-guided construction of synthetic gene networks with predicted functions. Nat Biotechnol 2009, 27:465-471.

4. Cantone I, Marucci L, Iorio F, Ricci M, Belcastro V, Bansal M, Santini S, di Bernardo M, di Bernardo D, Cosma M: A yeast synthetic network for in vivo assessment of reverse-engineering and modeling approaches. Cell 2009, 137:172-181.

5. Danino T, Mondragon-Palomino O, Tsimring L, Hasty J: A synchronized quorum of genetic clocks. Nature 2010, 463:326-330.

6. Becskei A, Seraphin B, Serrano L: Positive feedback in eukaryotic gene networks: cell differentiation by graded to binary response conversion. EMBO J 2001, 20:2528-2535.

7. Koch AJ, Meinhardt H: Biological pattern formation: from basic mechanisms to complex structures. Rev Mod Phys 1994, 66:1481-1507.

8. Hartmann D: Pattern formation in cultures of Bacillus subtilis. J Bio/ Syst 2004, 12:179-199.

9. Reeves GT, Muratov CB, Schupbach T, Shvartsman SY: Quantitative models of developmental pattern formation. Develop Cell 2006, 11:289-300.

10. Basu S, Gerchman Y, Collins CH, Arnold FH, Weiss R: A synthetic multicellular system for programmed pattern formation. Nature 2005 434:1130-1134.

11. Isalan M, Lemerle C, Serrano L: Engineering gene networks to emulate Drosophila embryonic pattern formation. PLOS Biol 2005, 3:488-496.

12. Goldbeter A: Computational approaches to cellular rhythms. Nature 2002, 420:238-245.

13. Elowitz MB, Leibler S: A synthetic oscillatory network of transcriptional regulators. Nature 2000, 403:335-338.

14. Atkinson MR, Savageau MA, Myers JT, Ninfa AJ: Development of genetic circuitry exhibiting toggle switch or oscillatory behavior in Escherichia coli. Cell 2003, 113:597-607.

15. Becskei A, Serrano L: Engineering stability in gene networks by autoregulation. Nature 2000, 405:590-593.

16. Lewis J: Autoinhibition with transcriptional delay: a simple mechanism for the Zebrafish somitogenesis oscillator. Curr Bio/ 2003, 13:1398-1408.

17. Bratsun D, Volfson D, Tsimring LS, Hasty J: Delay-induced stochastic oscillations in gene regulation. Proc Natl Acad Sci USA 2005, 102:14593-14598.

18. Mather W, Bennett MR, Hasty J, Tsimring LS: Delay-induced degradeand-fire oscillations in small genetic circuits. Phys Rev Lett 2009, 102:068105.

19. Isaacs FJ, Hasty J, Cantor CR, Collins JJ: Prediction and measurement of an autoregulatory genetic module. Proc Natl Acad Sci USA 2003, 100:7714-7719.

20. McMillen D, Kopell N, Hasty J, Collins JJ: Synchronizing genetic relaxation oscillators by intercell signaling. Proc Natl Acad Sci USA 2002, 99:679-684.

21. Garcia-Ojalvo J, Elowitz MB, Strogatz SH: Modeling a synthetic multicellular clock: Repressilators coupled by quorum sensing. Proc Natl Acad Sci USA 2003, 101:10955-10960.

22. Stricker J, Cookson S, Bennett MR, Mather WH, Tsimring LS, Hasty J: A fast, robust and tunable synthetic gene oscillator. Nature 2008, 456:516-519.

23. Kuhlman T, Zhang Z, H SJM, Hwa T: Combinatorial transcriptional control of the lactose operon of. Escherichia coli 2007, 104:6043-6048.
24. Purnick PE, RW: The second wave of synthetic biology: from modules to systems. Nat Rev Mol Cell Biol 2009, 10:410-422

25. Wilkinson DJ: Stochastic modelling for quantitative description of heterogeneous biological systems. Nat Rev Genet 2009, 10:122-133.

26. Hersch GL, Baker TA, Sauer RT: SspB delivery of substrates for CIpXP proteolysis probed by the design of improved degradation tags. Proc Natl Acad Sci USA 2004, 101:12136-12141.

27. Wong WW, Tsai TY, Liao JC: Single-cell zeroth-order protein degradation enhances the robustness of synthetic oscillator. Mol Syst Biol 2007, 3:130

28. Narang A: Effect of DNA looping on the induction kinetics of the lac operon. J Theor Biol 2007, 247:695-712.

29. Bernstein J, Khodursky A, Lin PH, Lin-Chao S, Cohen S: Global analysis of mRNA decay and abundance in Escherichia coli at single-gene resolution using two-color fluorescent DNA microarrays. Proc Nat/ Acad Sci USA 2002, 99:9697-9702.

30. Dong $Y H$, Xu JL, Li XZ, Zhang LH: AiiA, an enzyme that inactivates the acylhomoserine lactone quorum-sensing signal and attenuates the virulence of. Erwinia carotovora 2000, 97:3526-3531.

31. Verdugo A, Rand R: Hopf bifurcation in a DDE model of gene expression. Commun Nonlinear Sci Numer Simul 2008, 13:235-242.

32. Wang $L H$, Weng $L X$, Dong $Y H$, Zhang $L H$ : Specificity and enzyme kinetics of the quorum-quenching $\mathrm{N}$-acyl homoserine lactone lactonase (AHLlactonase). J Biol Chem 2004, 279:13645-13651.

33. Nikaido $H$, Rosenberg EY: Effect of solute size on diffusion rates through the transmembrane pores of the outer membrane of Escherichia coli. $J$ Gen Physiol 1981, 77:121-135.

34. Murray JD: Mathematical Biology: I. An Introduction New York: Springer Verlag; 2002.

35. Canton B, Labno A, Endy D: Refinement and standardization of synthetic biological parts and devices. Nat Biotechnol 2008, 26:787-793.

\section{doi: 10.1186/1752-0509-4-48}

Cite this article as: Rodrigo et al., Robust dynamical pattern formation from a multifunctional minimal genetic circuit BMC Systems Biology 2010, 4:48

\section{Submit your next manuscript to BioMed Central and take full advantage of:}

- Convenient online submission

- Thorough peer review

- No space constraints or color figure charges

- Immediate publication on acceptance

- Inclusion in PubMed, CAS, Scopus and Google Scholar

- Research which is freely available for redistribution 